\title{
Immunoliposomes: A Multipurpose Strategy in Breast Cancer Targeted Therapy
}

\author{
Enrique Barrajón-Catalán, María P. Menéndez-Gutiérrez, Alberto Falcó, \\ Miguel Saceda, Angela Catania and Vicente Micol \\ Institute of Molecular and Cell Biology (IBMC), Miguel Hernández University, \\ Spain
}

\section{Introduction}

One of the main challenges in chemotherapy is the delivery of effective doses of cytotoxic agents to the tumor site and simultaneously to minimize the side effects on normal cells. The use of drug delivery systems (DDS) can improve the pharmacological properties of many agents, modifying their pharmacokinetics and biodistribution.

Among the DDS available, liposomes are one of the most used and promising. Cytotoxic drug incorporation into liposomes has been reported since 1970. Thanks to their special structure, they are able to encapsulate both hydrophilic and hydrophobic compounds. Today several drugs with various chemical properties (hydrophilic drugs such as N(phosphoacetyl)-L-aspartate or hydrophobic drugs such as doxorubicin) have been succesfully encapsulated into liposomes. Compared to conventional chemotherapeutic drugs (non-encapsulated), the encapsulation of these drugs into liposomes shows several advantages, such as a considerable reduction of side effects associated to conventional chemotherapy, increase of solubility, improvement of therapeutic index and prolonged duration of exposure (Papahadjopoulos, Allen et al., 1991; Drummond, Meyer et al., 1999). Moreover, tumours are one of the primary sites for accumulation of liposomes, where they concentrate due to the higher permeability of the vascular endothelium surrounding tumours. However, despite their unquestionable advantages, unfortunately, liposomes are non site-specific drug delivery systems.

Antibody based targeted therapies are the most promising strategies to improve the selectivity and potency of current cancer treatments. The increasing number of new anticancer drugs based on antibodies demonstrates this assumption (Sznol \& Holmlund, 1997; Reichert, Rosensweig et al., 2005). However, selectivity provided by the use of antibodies can also be used, not only for direct treatment but to target other anticancer drugs. In this sense, the conjugation of complete or fragmented antibodies to liposomes has resulted in the next generation of drug delivery systems, i.e. immunoliposomes (Park, Hong et al., 1997; Noble, Kirpotin et al., 2004). In this strategy, the liposome acts as a drug carrier, and the antibody allows bringing the drug system specifically to its target.

Tumour cells have few specific molecules for recognition as they share many common features with normal cells. Then, the main issue when designing selective immunoliposomes is to find a proper target. Since complete selectivity is hard to achieve, the target must be as selective as possible, i.e. by using tumor-overexpressed proteins as 
targets. These proteins increase their expression levels up to 100 times compared to normal cells, and are suitable to be used as best target proteins (Barrajón-Catalán, MenéndezGutiérrez et al., 2010).

In this chapter, two promising targets are presented as examples of the immunoliposomes versatility and their usefulness in breast cancer models. The first one is the epithelial cell adhesion molecule (EpCAM), which is overexpressed in carcinomas of several origins including breast carcinomas (Armstrong \& Eck, 2003; Went, Lugli et al., 2004). The second one is the human epidermal growth factor receptor 2 (HER2), which is overexpressed in 20$30 \%$ of breast and ovarian cancers carrying a bad prognosis (Barrajón-Catalán, MenéndezGutiérrez et al., 2010). Currently, several antibody-derived drugs based on these targets are already in use in cancer clinical therapies. For instance, Edrecolomab and Trastuzumab are antibodies against these proteins which are used in cancer therapies (Punt, Nagy et al., 2002; Reichert, Rosensweig et al., 2005).

The diversity of chemotherapeutic drugs vehiculized into immunoliposomes is continuously growing, as new drugs or natural compounds are incorporated to the anticancer therapeutic arsenal (Mamot, Drummond et al., 2003; Yang, Choi et al., 2007; Yuji Yamamoto, 2011 Jan). Alternatively, other new strategies based on the use of immunoliposomes, but including lytic peptides, are focused on specifically promoting the lysis of cancer cells (Barrajón-Catalán, Menéndez-Gutiérrez et al., 2010). In this regard, melittin represents one of the most studied lytic peptides, and it has shown antiinflammatory and anticancer activities and low toxicity, and it has been used in several therapeutical approaches (Son, Lee et al., 2007; Soman, Baldwin et al., 2009).

This chapter is focused on the latest mentioned type of immunoliposomes, the so-called lytic immunoliposomes (LILs), which might become a fast and specific new treatment for breast cancer. The feasibility of LILs carrying melittin and containing antibodies against the two above mentioned targets, EpCAM and HER2, is shown. These immunoliposomes have been used to treat a panel of human breast cancer cells bearing different EpCAM or HER2 expression levels. In both cases, the morphological changes observed in treated cancer cells suggested a cytolytic process, as it could be expected given melittin lytic properties.

The approach presented here may suppose a specific, versatile and effective strategy for the treatment of tumors overexpressing a surface antigen. The incorporation of new antibodies to this model will allow targeting additional molecular sites in cancer cells, which will surely provide treatments for cancer therapeutics with increased selectivity and lower side effects and, it will probably contribute to decrease the appearance of resistances.

\section{Preliminary aspects to be considered}

\subsection{The molecular target}

As stated above, the most difficult task when designing immunoliposomes is choosing the right molecular target. Since complete selectivity is hard to achieve, the therapy must be as specific as possible in order to reduce side effects and to increase the potency of the model.

To obtain optimal results, the target must fulfil the following conditions:

- It must be a surface antigen and has to be located in an accessible domain of the cytoplasmic membrane.

- It must be as much antigenic as possible in order to produce the corresponding antibody. 
- $\quad$ Proteins are preferred rather than other antigens such as carbohydrates.

- Tumor specific antigens are preferred, but these are rare. Then, tumor overexpressed antigens are a more accessible alternative. The higher the overexpression level is, the more selective the system gets.

Finding the perfect target is unlikely, but there are several promising candidates. The most used and characterized antigens belong to the HER family. Moreover, other targets have been used and well characterized: EpCAM in breast and gastric cancers (Cimino, Halushka et al., 2009; Wenqi, Li et al., 2009), PSMA in prostate cancer (Ikegami, Tadakuma et al., 2005; Ikegami, Yamakami et al., 2006) or MUC1 in colon and prostate cancer (McDermott, Crocker et al., 2001; Papadopoulos, Sivridis et al., 2001).

The continuous discovery of new tumor specific and/or tumor overexpressed antigens by many research groups working in this field will enormously contribute to the development of these treatments, by generating a constant increase in the number of potential antibodies. Moreover, new advances in proteomic investigation will also help to establish successful therapies (Conrotto, Roesli et al., 2008; Madoz-Gúrpide, Kuick et al., 2008).

\subsection{The immunoliposome design}

The linking of antibodies to liposomes has been intensely studied from different perspectives (see review (Hansen, Kao et al., 1995)). Most of these techniques have been used in different cellular and animal models. Since every case may have specific requirements, the linking technology is normally chosen depending on the availability of the chemical groups involved, such as lipid polar head groups, linker's nature and antibody characteristics.

Once the technique is selected, the right linker will be chosen according to the procedure. The most common linkers are modified lipids containing the maleimide moiety. On the other hand, the antibody must be derivatized in order to be linked to the lipid moieties. One of the most common derivatization techniques uses Traut's reagent, which yields thiol groups in the antibody, what allows the subsequent reaction with the maleimide group of the modified lipids through a covalent bonding (Kirpotin, Park et al., 1997).

Regardless the selected protocol, there are some aspects to take into account when immunoliposomes are designed:

- Immunogenicity of the final product must be as low as possible, and the drug must remain in blood circulation as long as possible. Pegylation solves both aspects in a smart way. First, the use of pegylated lipids into liposomes at $1-5 \%$ molar percentage increases blood permanence time because it reduces uptake rate by the reticuloendothelial system. Second, it decreases the immunogenicity of the preparation as the liposomes become "invisible" to the immune system due to the polyethylene glycol (PEG) tails (Petrak, 2005; Elbayoumi \& Torchilin, 2006). In addition, pegylated liposomes show a superior tumor delivery capacity compared with conventional liposomes composed of natural phospholipids (Allen \& Cullis, 2004). Although there are different PEG lengths, PEG-2000 is the most used in pharmacological preparations (Sadzuka, Kishi et al., 2003).

- Lipid composition is also very important because it determines the fluidity of the liposomal membrane, and therefore, its ability to encapsulate or retain compounds. For example, the presence of cholesterol increases the rigidity of the liposome membrane, contributing to the encapsulation improvement of hydrophilic compounds into the inner aqueous cavity. When hydrophobic molecules are used, the lipid composition is 
also an important issue, because it must provide an adequate environment, capable to retain hydrophobic molecules in the required active conformation and the ability to transfer them to target membranes (Maruyama, Kennel et al., 1990).

- Finally, the antibody attached to the liposome can be used either as a whole protein or fragmented. Several approaches have been developed using the single-chain antibody variable region (scFv) (Mamot, Drummond et al., 2003; Hu, Chen et al., 2006), the complete variable region (Fab) (Kirpotin, Drummond et al., 2006) or the whole antibody (Barrajón-Catalán, Menéndez-Gutiérrez et al., 2010). It must be considered that, scFv and Fab (Figure 1) are less immunogenic than the whole antibody, however these fragments are not easy to obtain. The production of Fab requires a large amount of the original antibody and the protocol includes its digestion by pepsin or other enzymes. In contrast, the use of $\mathrm{scFv}$ fragments requires cloning designs and recombinant production systems.

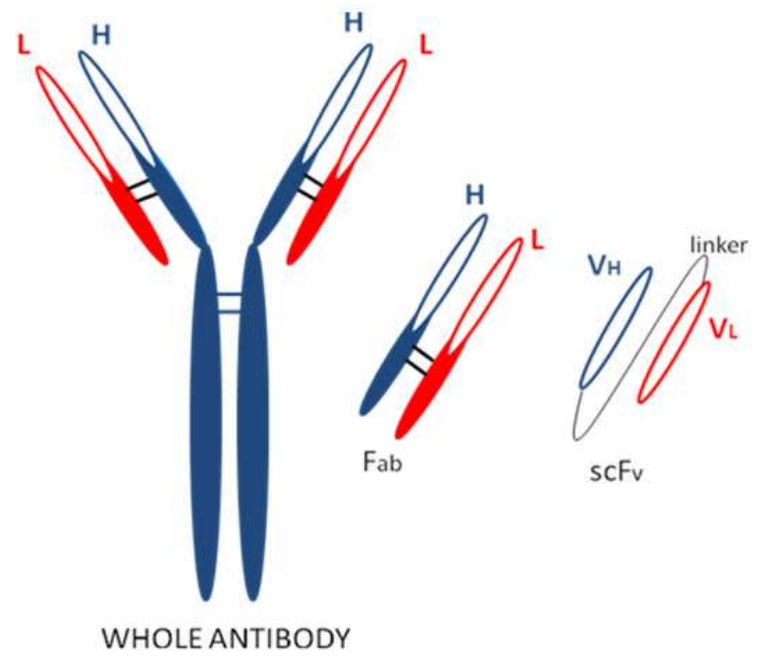

Fig. 1. Different classes of antibody derivatives used for immunoliposome synthesis. Heavy chain $(\mathrm{H})$ is shown in blue and light chain $(\mathrm{L})$ in red, variable region appears as empty ellipses. Whole antibody (on the left) can be used to obtain the Fab fragment with constant and variable region linked by disulfide bonds. scFV fragment contains only the variable regions of both heavy and light chains bound by a linker.

\subsection{The lytic peptides}

Antimicrobial peptides are gene encoded natural peptide antibiotics being part of the innate defences of many organisms (Patrzykat \& Douglas, 2005). Among them, lytic peptides (LPs) are those showing highest cytolytic activity. Several studies have shown that LPs have cationic and amphipathic properties, which allow them to interact with lipid cell membranes of most pathogens, often negatively-charged (Lohner \& Blondelle, 2005), by forming pores or ion channels (Bechinger, 1997). Several models have been proposed to explain this mechanism, i.e. barrel-stave, carpet-like, toroidal pore formation or detergent-type micellization models (Allende, Simon et al., 2005). Regardless the molecular mechanism, LPs promote increased 
membrane permeabilization, leakage of cell content and osmotic instability, which leads to the unavoidable cell death. In some cases, peptide diffusion to intracellular targets has also been described. The capacity of LPs to promote cell lysis has been extensively studied in order to develop new anticancer approaches (Hoskin \& Ramamoorthy, 2008).

LPs can be included in LILs as active molecules that promote cytotoxic effects. LPs have already been used covalently linked to hormone segments in order to increase their efficacy and selectivity on several types of cancer cells (Gawronska, Leuschner et al., 2002; Hansel, Enright et al., 2006). Nevertheless, a therapy based on this approach might be valid only for those tumors expressing hormone receptors, such as prostate or breast cancers. However, LILs may be applied to any kind of tumors. For instance, previous reports have shown the activity of LILs against hepatoma cancer cells (Hu, Chen et al., 2006).

Among the LPs derived from insects and amphibians, melittin, from bee venom, has recently shown anticancer properties but its precise mechanism of action is still uncertain (Son, Lee et al., 2007). Melittin is a 26 amino acid linear peptide (GIGAVLKVLTTGLPALISWIKRKRQQ) with alpha-helix conformation. The distribution of its amino acid sequence is the main responsible of the amphipathic nature of this peptide: the amino-terminal region (residues 1-20) is predominantly formed by hydrophobic residues, whereas the carboxy-terminal region (residues 21-26) is hydrophilic due to the presence of a tandem of basic amino acids (Raghuraman \& Chattopadhyay, 2007). Melittin has been the LP choice in several studies using LILs. Among the reasons for this are: its particular amphipatic nature, which allows it to bind to lipid membranes with high affinity, its activity against tumor cells, its relatively low market value and its availability. It is also water soluble, which facilitates its manipulation.

Although melittin molecular mechanism seems to be controversial, the most accepted model proposes that melittin destroys target membranes by pore formation and destabilization through the barrel-stave mechanism (Sui, Wu et al., 1994; Lin \& Baumgaertner, 2000). In this sense, once LIL binds to targeted cell, a lytic process occurs in a few hours with no signal of apoptosis (Barrajón-Catalán, Menéndez-Gutiérrez et al., 2010).

To fully characterize LILs model, the selected LP must be adequately quantified. For this task, HPLC coupled to fluorescent, diode array or mass detection is the most commonly used method (Perez-Paya, Braco et al., 1991; Barrajón-Catalán, Menéndez-Gutiérrez et al., 2010) but also capillary chromatography (Pacakova \& Stulik, 2000) can be utilised.

\subsection{The cellular model}

The choice of a suitable cellular model is as important as the target to test the efficacy of the LIL system. Several cell lines showing gradual differences in the expression levels of the targeted protein may be required for these studies in order to make sure that cytotoxic effects are dependent on the antigen expression. Thus, one of the preliminary tasks is the quantification of the expression levels of the selected cellular lines. Nevertheless, it must be checked that all the quantified protein is located on the cell membrane. In some cases, a significant percentage of the overexpressed protein shows an intracellular location, and then, this protein is obviously not suitable to be used as target for LILs. Fortunately, many breast cancer cellular lines are readily available and well characterized, facilitating this task. When selecting cell lines, to assess their in vivo tumorogenic capacity becomes also an important issue. One of the critical steps in preclinical investigations with LILs and other pharmaceutical formulations, is to prove their in vivo activity against xenotransplanted 
cancer cells in athymic mice. Not all cells are able to establish and grow in these mice on their own. For example, some breast cancer cells present strogen-dependent growth (Saceda, Knabbe et al., 1991) and estradiol must be used as coadjuvants in order to obtain successful in vivo tumourogenic activity. On the other hand, other cells need the use of polymeric coadjuvants, such as matrigel, to be implanted in mice and the tumor cells must be coinjected with this polymer.

\section{Lytic immunoliposome models}

\subsection{EpCAM immunoliposomes}

EpCAM is a transmembrane protein (Armstrong \& Eck, 2003; Went, Lugli et al., 2004; Trzpis, McLaughlin et al., 2007) which mediates epithelium-specific $\mathrm{Ca}^{2+}$ independent cellcell adhesions. EpCAM is overexpressed in carcinomas of several origins such as breast, gastric, colorectal, prostate and liver (Went, Lugli et al., 2004; Wenqi, Li et al., 2009). EpCAM antigen has been used in immunotherapy studies (Braun, Hepp et al., 1999; Winkler, MartinKillias et al., 2009) against breast cancer cells with good results. There is a commercial antiEpCAM antibody called Edrecolomab (Panorex ${ }^{\circledR}$ ), which is currently being used in the treatment of colorectal, breast and gastric cancers (Braun, Hepp et al., 1999; Haller, 2001). Due to these reasons, EpCAM supposes an attractive and promising target to be challenged through the use of lytic immunoliposomes therapy.

In this first study, anti-EpCAM coupled immunoliposomes were used to target human cancer cells in vitro. Large unilamellar vesicles were sterically stabilized by using a pegylated phospholipid (Kirpotin, Park et al., 1997) and then, covalently coupled to a commercial anti-EpCAM antibody using a phospholipid linker. Subsequently, pure melittin was incorporated into the immunoliposome mixture. Unbonded peptide was separated by gel filtration chromatography. For the complete protocol, see reference (Barrajón-Catalán, Menéndez-Gutiérrez et al., 2010).

Once immunoliposomes were obtained, the expression levels of EpCAM were analyzed in the cell lines. In this case, the selected cell lines were MCF7 and MDA-MB231, both derived from a pleural effusion of a human breast adenocarcinoma; MCF7 is documented to have high expression levels of EpCAM. On the contrary, MDA-MB231 shows a very low expression (Prang, Preithner et al., 2005). To ensure this point, EpCAM expression was analyzed by Western blot using an anti-EpCAM antibody (Figure 2) in both cell lines, confirming the previously published results.

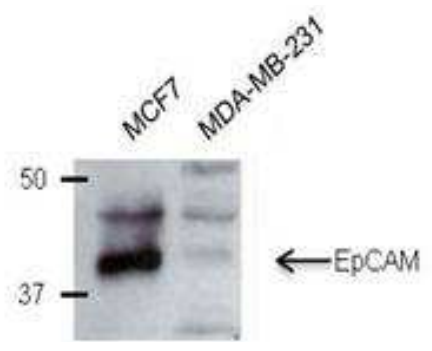

Fig. 2. Anti-EpCAM Western blot showing the expression levels of the protein in MCF7 and MDA-MB231 cells. Molecular weight references (in $\mathrm{kDa}$ ) are shown in the left side of the picture. 
To ensure that targeted antigen is mainly expressed as a surface protein instead of being located in endosomes or vacuoles, and that it can be reached by the antibodies linked to the LILs, fluorescence-activated cell sorting (FACS), a type of flow cytometry, can be used. Membrane expression levels of surface antigens can be measured and compared using this sensitive technique. Other alternative is to perform Western blot analysis of membrane protein extracts obtained after subcellular fractionation. One example of the results obtained by FACS is shown in figure 3, where EpCAM membrane expression levels in MCF7 and MDA-MB231 cell lines are compared.

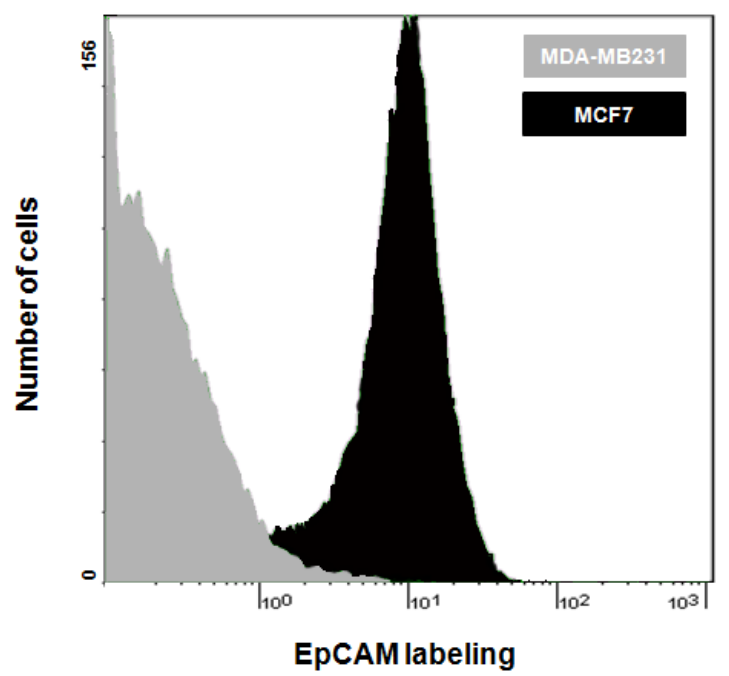

Fig. 3. Analysis of EpCAM expression on cell membranes by FACS in MDA-MB231 (grey) and MCF7 (black).

Once the expression levels of the chosen antigen have been determined in both cell lines, binding of immunoliposomes to the cells can be assessed by fluorescence microscopy. For this purpose, the lipid mixture can be labeled using a fluorescent phospholipid conjugated with rhodamine. Alternatively, fluorescein isothiocyanate (FTIC) conjugated antibodies can also be used. In the present example, images of two cell lines were obtained after treatment with fluorescent immunoliposomes, which were labeled both with rhodamine conjugated phosphatidylethanolamine (PE) and anti-EpCAM FTIC-conjugated (Figures 4A and 4B). MCF7 cells, which overexpress EpCAM antigen, present a double labeling due to the binding of complete immunoliposomes (red of rhodamine-PE and green of FITC-labeled antibody). However, MDA-MB231 cells showing low EpCAM expression levels present only a weak red fluorescence due to the background signal deriving from rhodamine-labeled liposomes lacking the antibody. The cytotoxic activity of the LILs can be also analyzed using fluorescence microscopy or flow cytometry. Some probes, such as DAPI are able to penetrate only in permeabilized cells or cells with damaged membranes, then these probes are specific for cell death. The efficacy of the cytotoxic effect of LILs may be also monitorized using DAPI fluorescence because of the effect of lytic peptides on cell membrane (Figure 4C). MCF7 cells show a triple-labeling (red from lipids, green from antibody and blue from DAPI), indicating that the binding of the LILs to the targeted cells is followed by cell death. 

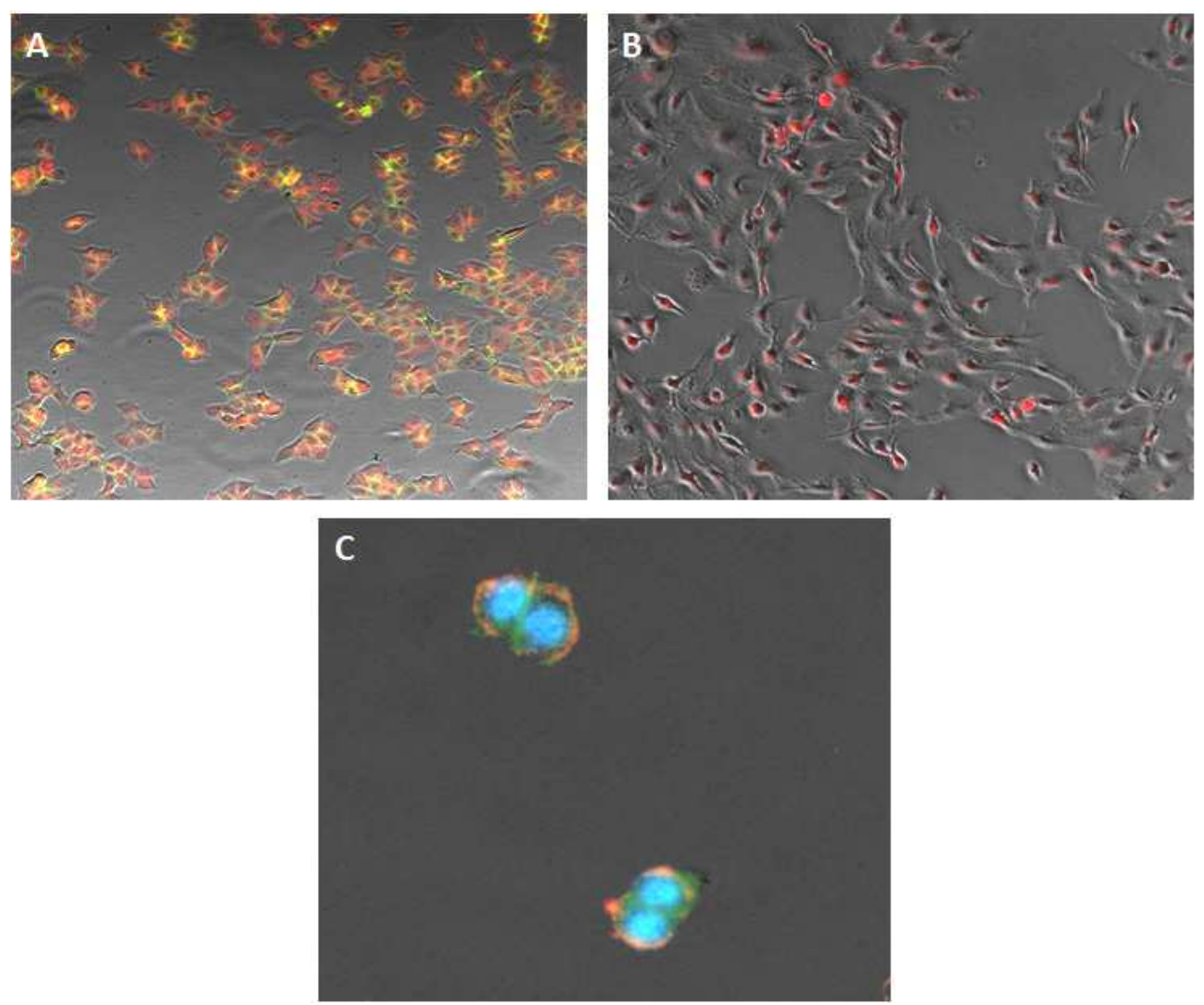

Fig. 4. Fluorescence microscopy imaging of the treatment of breast cancer cells with LILs containing EpCAM antibody. MCF7 (A) and MDA-MB231 (B) cells treated with LILs carrying rhodamine conjugated PE and anti-EpCAM FTIC conjugated antibody. (C) MCF7 cells treated with the same LILs but in the presence of DAPI (blue fluorescence).

Fluorescent microscopy imaging is a useful technique for obtaining qualitative results, nevertheless quantification of the binding and cytotoxic effects of LILs are key aspects to consider in order to test LILs efficacy. The use of flow cytometry is a great approach to quantify these two aspects simultaneously. To achieve this goal, LILs carrying FITCconjugated EpCAM antibody were used to treat both cell lines and then propidium iodine, which only penetrates permeabilized cells, was added to monitorize cell death. Figure 5 shows the treatment of MDA-MB231 and MCF-7 cells using labeled LILs and propidium iodine. The whole bar indicates the percentage of EpCAM FITC labeled cells and stripped bar shows dead cells (propidium iodine labeled), which are EpCAM positive, i.e. cells showing double labeling. EpCAM overexpressing MCF7 cells treated with LILs showed ten times more fluorescence, due to antibody labeling, than MDA-MB231 cells, which showed a low EpCAM expression level (Figure 5). This indicates that MCF7 cells were succesfully reached by LILs. Furthermore, only MCF7 cells were labeled with propidium iodine, indicating that the binding of LILs to these cells was followed by permeabilization and cell death. This confirms previous fluorescence microscopy results. 


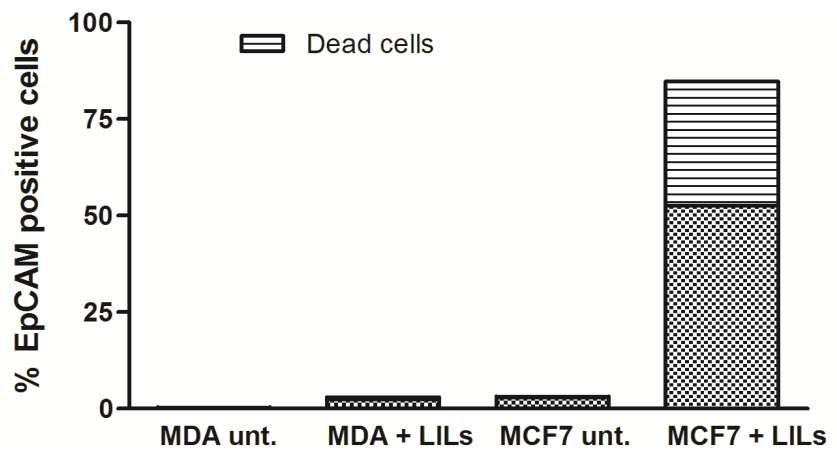

Fig. 5. Flow cytometry results of MDA-MB231 and MCF-7 cells treated with LILs carrying EpCAM antibody and propidium iodine. Total bars indicate the percentage of EpCAM positive-expressing cells while stripped fraction of the bars indicates the percentage of these cells reached by propidium iodine.

Other techniques to quantify the effects of LILs on cancer cells are those focused to determine cell viability. MTT (Barrajon-Catalan, Fernandez-Arroyo et al., 2010) and violet crystal are among the techniques most commonly used for detecting cell survival. Adequate control experiments must also be established in cell survival assays in order to obtain conclusions about LILs specificity. Target cells must be treated comparatively, using identical concentrations of either complete LILs (LILs carrying labeled antibody and lytic peptide), with immunoliposomes lacking the lytic peptide (LIP), or with liposomes bearing the lytic peptide but lacking the antibody (LIP + LP) (Figure 6). Although some level of unspecific cytotoxicity was found in the cells showing low antigen expression levels (MDAMB231), LILs exerted a strong cytotoxic effect on cells expressing high levels of EpCAM, which demonstrates LILs selectivity.

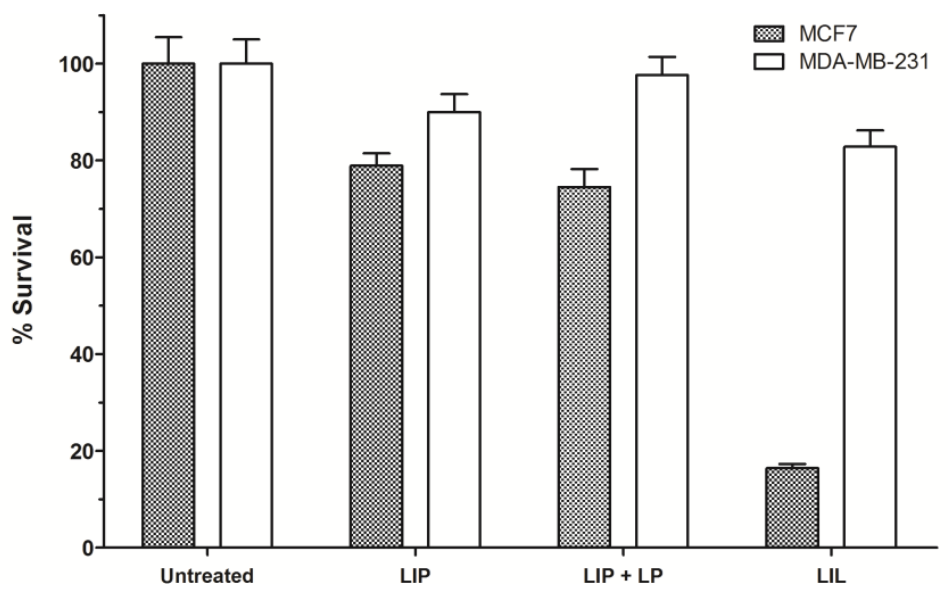

Fig. 6. Survival plots for two breast cancer cell lines having different EpCAM expression levels treated with various liposomal systems. Percentage of surviving cells was measured by MTT assay. MCF7 (dotted bars) and MDA-MB231 cells (white bars) were challenged with different constructs of liposomes and LILs. 


\subsection{HER2 immunoliposomes}

HER2 molecule is probably the most studied and clinically used target in anticancer studies involving immunotherapies. HER2 (or ErbB2) is a protooncogene belonging to the epidermal growth factor receptor family (EGFR or ErbB) of receptor tyrosine kinases (RTK) (Olayioye, Neve et al., 2000). HER2 is overexpressed in 20-30\% of breast and ovarian cancers (Slamon, Clark et al., 1987). Moreover, in normal adult tissues, HER2 is present only at low levels in certain epithelial cell types (Press, Cordon-Cardo et al., 1990). Several anticancer therapies targeting ErbB receptors have been developed, and the humanized monoclonal antibody that binds to HER2 (Herceptin ${ }^{\circledR}$ or trastuzumab) is currently in clinical use as an effective treatment for HER2 positive breast cancer (Slamon, Leyland-Jones et al., 2001). Trastuzumab has been used in several therapeutical approaches coupled to liposomes in order to increase anticancer therapy selectivity. Anti-HER2 immunoliposomes have shown binding and internalization capacities in HER2-overexpressing cells (Kirpotin, Park et al., 1997), which leads to intracellular drug delivery. Moreover, the use of doxorubicin-loaded anti-HER2 immunoliposomes exhibited significantly better therapeutic results, in HER2overexpressing xenograft models, compared to other treatments (Park, Hong et al., 2002).

The cellular model presented above for EpCAM LILs was composed of two cell lines, but more complex models can be used when several cell lines with different antigen expression levels are available. Figure 7 shows the level of HER2 expression of the total cellular extracts
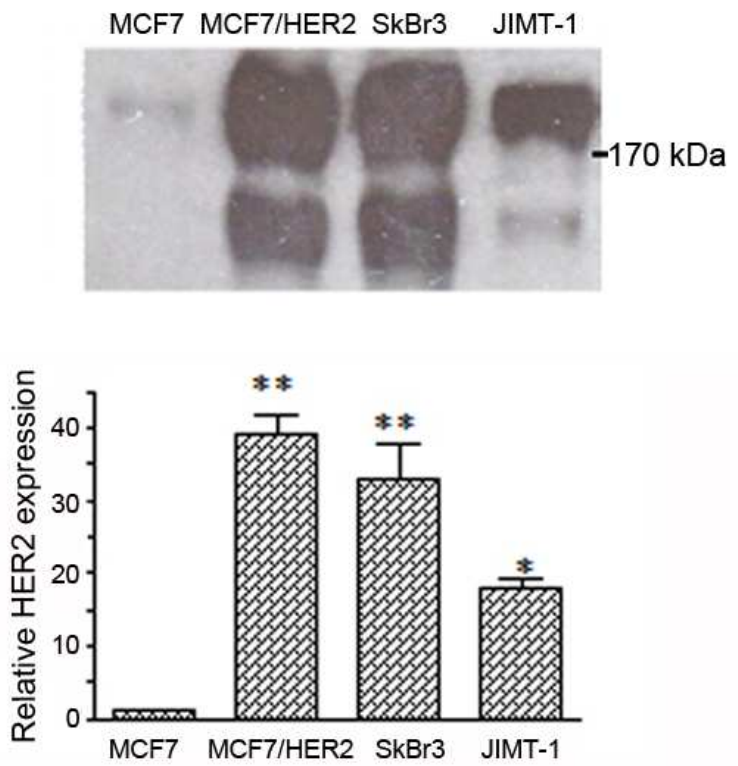

Fig. 7. HER expression level of four breast cancer cells by Western blot analysis. Top image shows the expression level in total cellular extracts from the different cell lines (MCF7, MCF7/HER2, SKBr3 and JIMT-1). Bottom image shows the results of the quantification of the bands using densitometric analysis. MCF7 and SKBr3 derive from a pleural effusion of a human breast adenocarcinoma, MCF7/HER2 stably overexpress HER2 after transfection and JIMT-1 derives from a human breast cancer clinically resistant to trastuzumab (Barrajón-Catalán, Menéndez-Gutiérrez et al., 2010). 
deriving from four different breast cancer cell lines (Barrajón-Catalán, Menéndez-Gutiérrez et al., 2010). Western blot analysis and quantification of the bands by densitometric analysis showed that MCF7/HER2 and SkBr3 exhibited the highest HER2 expression levels followed by JIMT-1 and MCF7 cells.

Nevertheless, to confirm the extracellular location of the antigen detected by Western blot, flow cytometry analysis using an antiboby able to detect only the antigen on the cell membrane was also performed. In this case, the different expression level of each cell line correlates to a displacement of the peak population to higher fluorescent levels, as shown in Figure 8 .
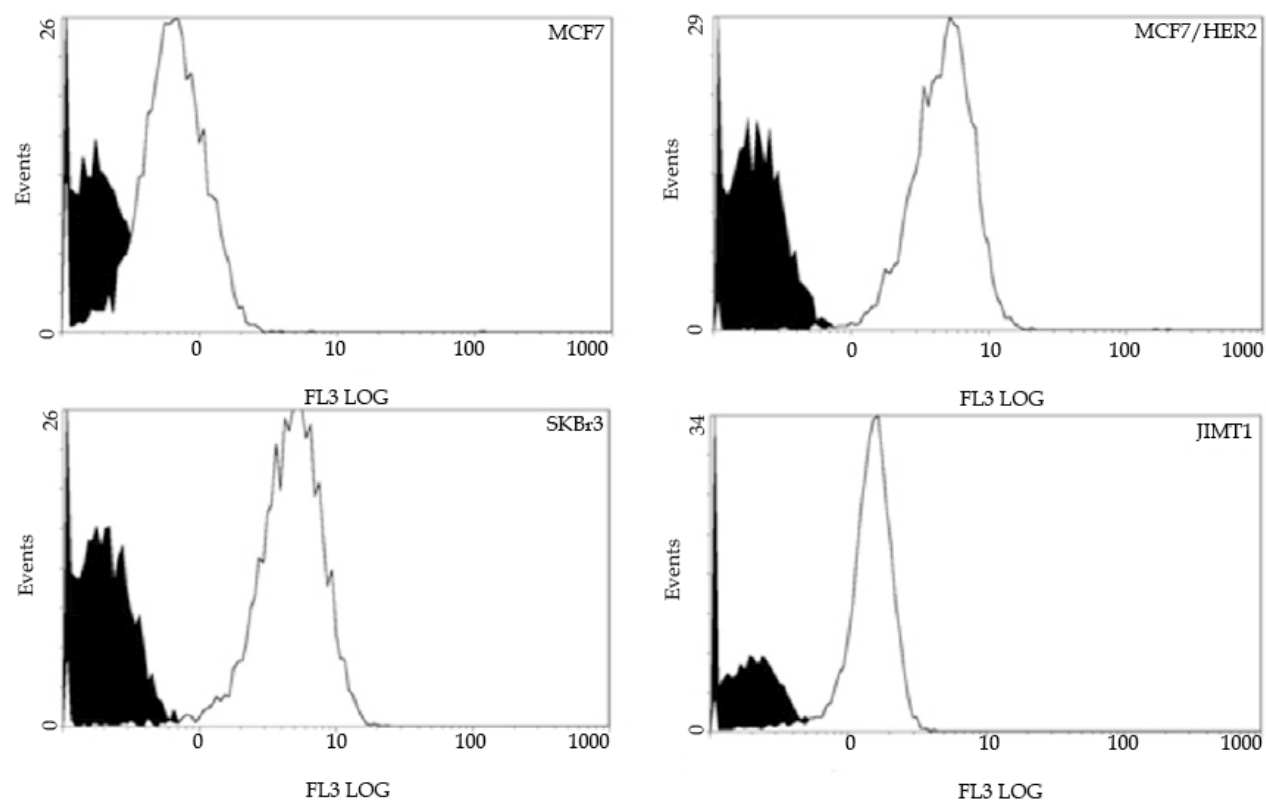

Fig. 8. FACS analysis of membrane HER2 expression in different breast cancer lines, named in the histograms.

The efficacy and selectivity of HER2-containing LILs on the different cell lines were tested in dose/response experiments using increasing concentrations of the LILs. Viability of MCF7 cells (with low HER2 expression) was the least affected by LILs. JIMT-1 cells viability was decreased in a higher degree than that one observed for MCF7 cells. Besides, MCF7/HER2 and SKBr3 cells, showing the highest levels of HER2 expression, underwent a sharp decrease of their viability at almost all the concentrations of the immunoliposomes preparation studied. Therefore, the higher the expression of HER2 was, the more active LILs system was (Figure 9A). Nevertheless, high concentrations of immunoliposomes would make the cytotoxic activity to be less cell-specific. Therefore, the biggest differences in cell viability were found at $0.3 \mathrm{mM}$ total lipid concentration (Figure 9B).

Flow cytometry experiments using specific probes for cell death are good complementary information to cell viability assays. However, new technical approaches as ImageStream analysis allow, not only the quantification of the fluorescence probes, but also obtaining in situ fluorescent images of the treated cells, when lipid or antibodies are labeled with 
fluorescent dyes. ImageStream-based analysis employs flow cytometry combined with microscopy and allows for statistical analysis of a variety of cellular parameters, as well as the visualization of cells in suspension during flow analysis via high-resolution bright-field, dark-field and fluorescence images (Zuba-Surma, Kucia et al., 2007).

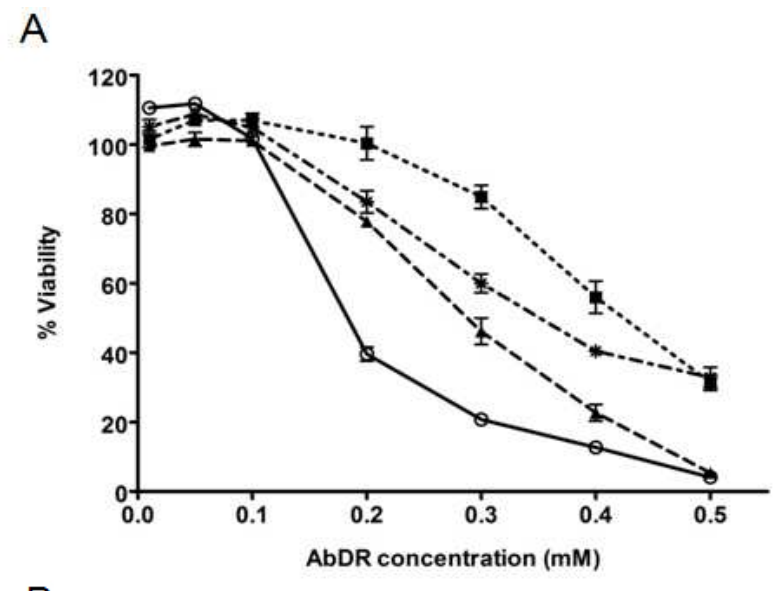

B

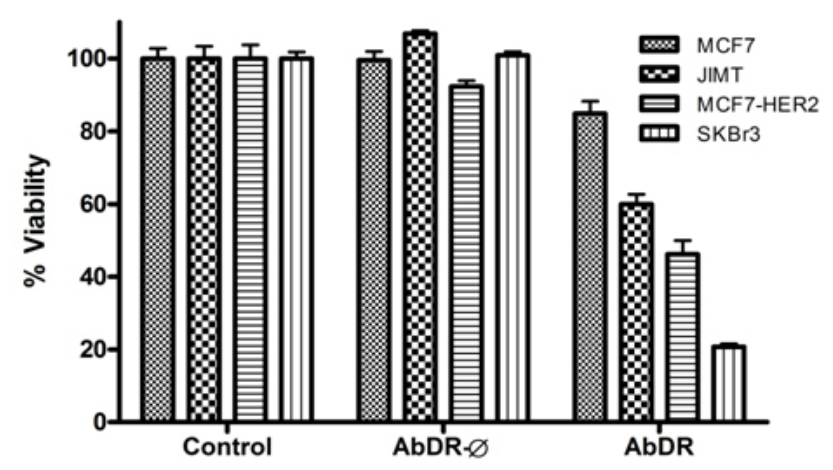

Fig. 9. Survival assays for HER2 LILs. (A) Plots showing the percentage of viable cells in each breast cancer cell line as determined by MTT assay after treatment with HER2 labeled LILs (-- -- JIMT-1, - $\mathbf{- 1}$ - MCF7/HER2, -o- SKBr3, - - - MCF7). (B) Percentage of viable cells determined by MTT assay for the four breast cancer cell lines after the treatment with 0.3 $\mathrm{mM}$ of LILs (AbDR) or immunoliposomes lacking the lytic peptide (AbDR- $\varnothing$ ).

Figure 10 shows the ImageStream assay results of the treatment of SkBr3 breast cancer cells with liposomes containing a rhodamine labeled lipid, but lacking antibody (Rhod-L, panel A) in comparison to labeled liposomes bearing HER antibody (Rhod-LILs, panel B) (Barrajón-Catalán, Menéndez-Gutiérrez et al., 2010). For quantification purposes, cell populations were separated in three different groups (subpopulations number 1, 2, and 3) attending to increasing fluorescence intensity signal. Figure 10 shows that Rhod-L (panel A) and Rhod-LILs (panel B) treated cells evidenced similar subpopulation 1 (those cells having lowest intensity and Rhod labeling). In contrast, Rhod-LILs treated cells showed a greater 
cell subpopulation 2 than Rhod-L treated cells (around double, i.e. $28 \%$ vs. $14 \%$ ) (see panels $\mathrm{A}$ and $\mathrm{B}$ and quantification in panel C). Moreover, Rhod-LILs treated cells showed another cell subpopulation, which supposed $5 \%$ of total cells (subpopulation 3, panel B), bearing a very strong Rhod labeling intensity, which was negligible in Rhod-L treated cells. The images corresponding to examples of sorted cells deriving from both treatments (Figure 10) clearly showed that subpopulations 2 and 3 exhibited a strong rhodamine labeling signal, which demonstrates the higher specificity of Rhod-LILs for SKBr3 cells. The total fluorescence in Rhod-LILs treated cells was approximately three times higher than that one of Rhod-L treated cells.

A

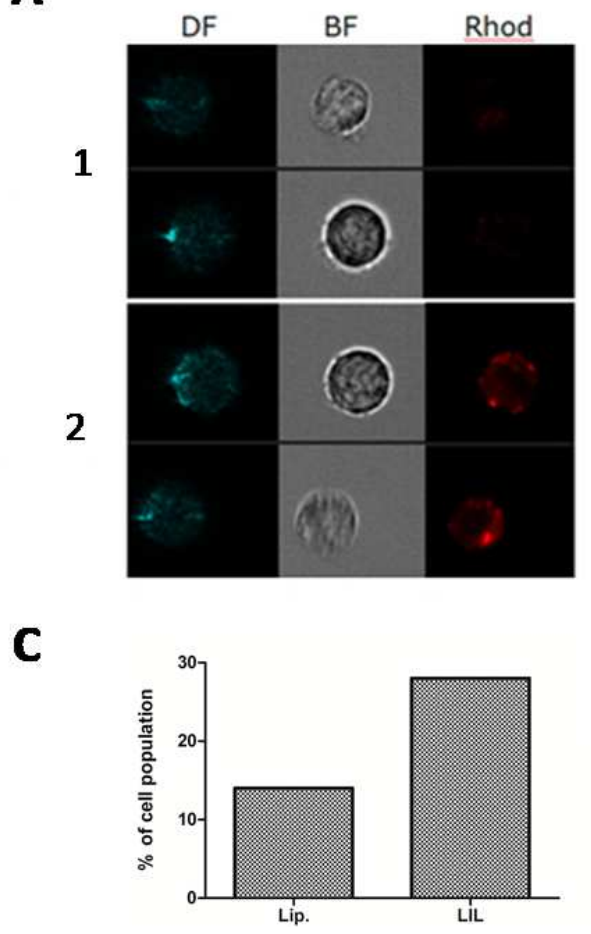

B
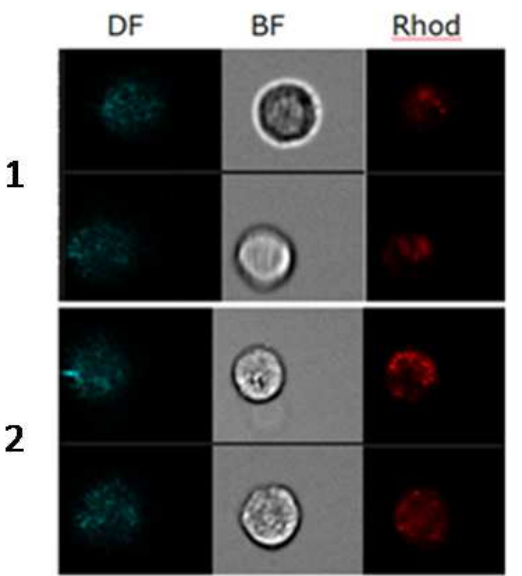

3

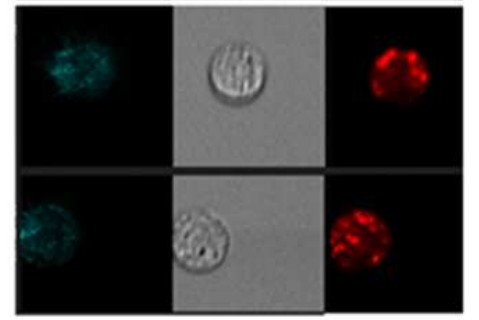

Fig. 10. (A and B) ImageStream results. SkBr3 breast cancer cells treated with liposomes containing a rhodamine labeled lipid, but lacking antibody (Rhod-L, panel A) in comparison to treatment with labeled liposomes bearing HER antibody (Rhod-LILs, panel B). 1, 2 and 3 are mean cell subpopulations having increasing fluorescence intensity signal derived from Rhod labeling. Composite images of dark-field (DF), bright-field (BF) and rhodamine fluorescence (Rhod) images are shown for both conditions. (C) Gated subpopulation 2 in Rhod-L and Rhod-LILs treated cells.

\subsection{LILs potential mechanism}

The cytotoxic effect of LILs on the tested breast cancer cells shown in these studies is quite fast. The main responsible molecule for this effect is the lytic peptide melittin, since immunoliposomes lacking melittin were devoid of such effect. Then it must be hypothesized 
that the cytotoxic action of LILs is mediated by a cytolytic process in which cells lysis is present. This event occurs within a few hours. Some descriptive images were taken to show this action (Figure 11). The figure clearly shows that cells died in a few hours period suffering a presumably lytic process, showing membrane cell disruption and leading to the release of the intracellular content followed by cell membrane retraction.
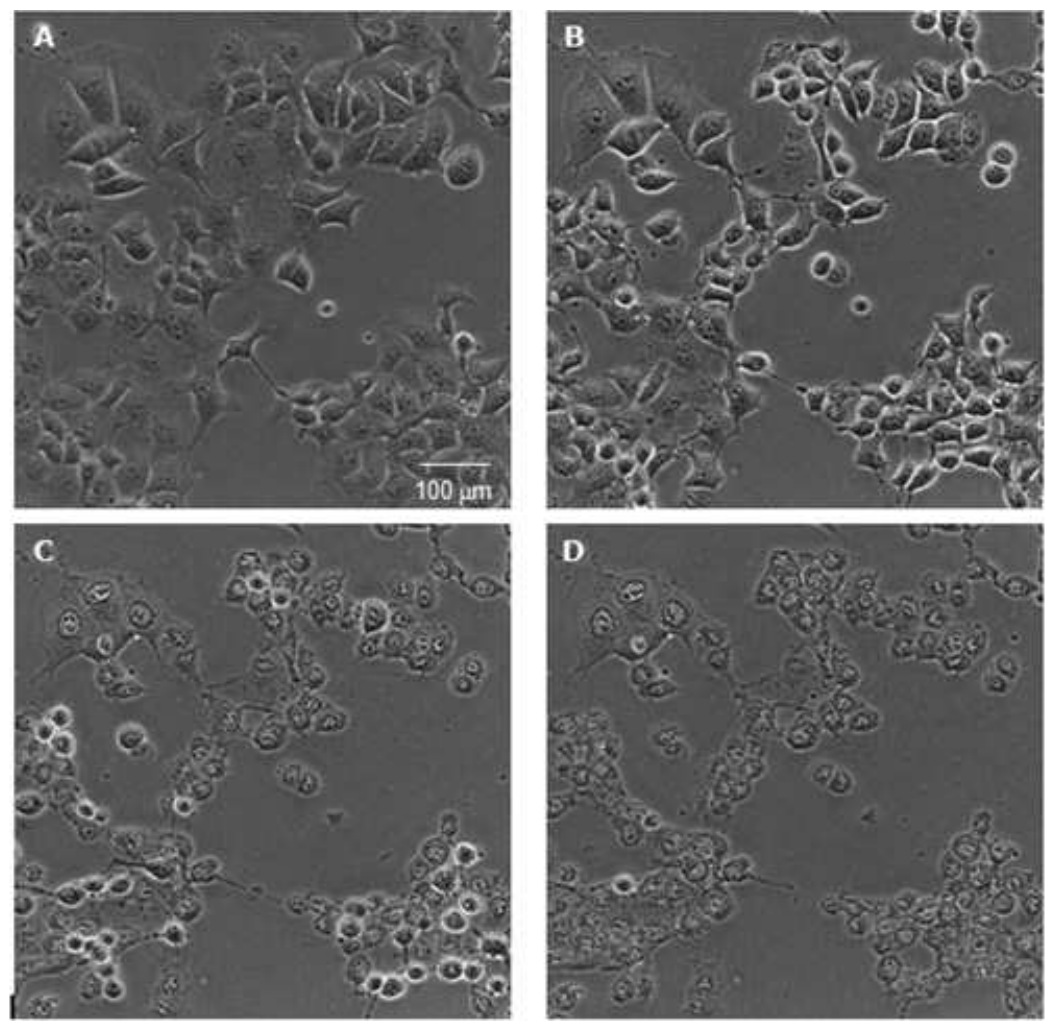

Fig. 11. Images from a LILs treatment. Images extracted from a time-course follow up experiment of the treatment of MCF7/HER2 cells for $4 \mathrm{~h}$ with LILs in a Nikon Eclipse TE2000U fluorescence microscope. The images were taken a 0 (A), 1 (B), 2 (C), and $4 \mathrm{~h}$ (D) using a $20 x$ lens.

Apparently, apoptosis is not directly implicated in the LILs mechanism as the time-lapse is too short (only a few hours) and no signals of DNA fragmentation or apoptotic bodies were observed after DAPI labeling (Figure 12).

LILs must be also structurally characterized by several techniques in order to test size and stability of the delivery system. Size, polydispersity and Z-potential can be obtained by Dinamic Light Scattering (Villari \& Micali, 2008), a potent and simple technique that allows to obtain these parameters without disrupting the LILs. The quantification of the amount of antibody present in LILs by Western blot is also useful in order to confirm the number of antibody molecules per liposome, which can give information about the efficacy of the derivatization of liposomes (Barrajón-Catalán, Menéndez-Gutiérrez et al., 2010). 

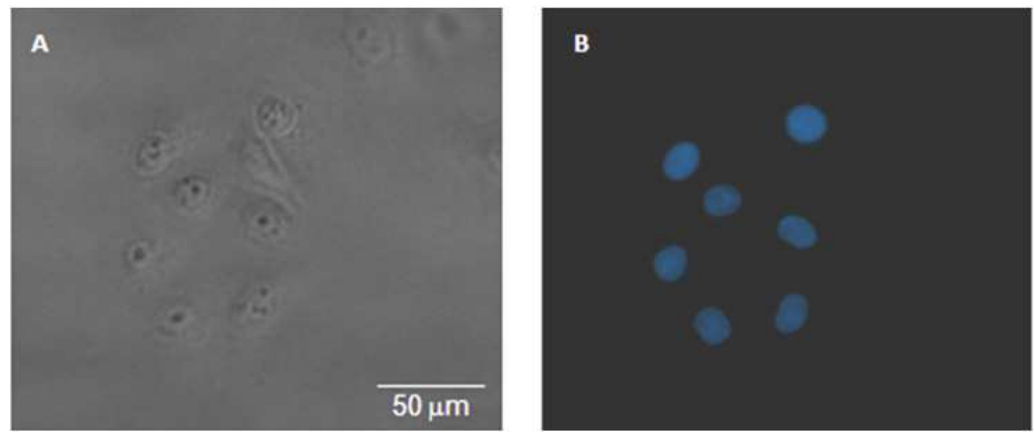

Fig. 12. DAPI labeling after LILs treatment. Contrast phase (A) and fluorescence images (B) of DAPI staining of MCF7-HER2 cells treated with LILs, images were taken using 40x lens.

\section{Conclusions}

LILs are a suitable pharmacological tool for the selective treatment of cancer cells. For their synthesis, liposomes containing an adequate lipid composition, and a choice of lytic peptides and antibody are required. LILs exhibit specific cytotoxicity against antigen overexpressing breast cancer cells. Moreover, this capacity correlates to the antigen expression level at the cell membranes. The morphological changes observed through microscopy, when cancer cells are treated, suggest a very fast mechanism of cell death, which might take place primarily through membrane pore formation involving a cytolytic process.

The approach presented here may suppose an specific and effective strategy for the treatment of tumors that show overexpression of a surface antigen, by using a low toxicity peptide such as melittin. The examples presented in this chapter describe the elements needed to synthesize lytic immunoliposomes and the techniques to assay their activity on cancer cells. Although these studies have been focused on cancer cells, if an overexpressed surface antigen exists, this approach may be extended to other cell types. Finally, immunoliposomes could be also used as delivery systems for other cytotoxic molecules, either from synthetic or natural origin, which could improve the selectivity or efficacy of these molecules on their cellular targets.

\section{Acknowledgments}

This investigation has been supported by Grant AGL2007-60778 and private funds provided by CGB-EDSON. We thank personnel of Elche University Hospital for their advice and help in the ImageStream analysis and for providing us with the commercial antibody. We also thank Dr. José A. Ferragut and Dr. Alfredo Carrato from IBMC, and Dra. Inmaculada Jiménez from Elche University Hospital and Dr. Jose Solla from Alicante University for their invaluable help. Figures 7-12 are reprinted from Cancer letters, 290, Barrajón-Catalán et al., Selective death of human breast cancer cells by lytic immunoliposomes: Correlation with their HER2 expression level, 192-203, with permission from Elsevier.

\section{References}

Allen, T. M. and P. R. Cullis (2004). "Drug delivery systems: entering the mainstream." Science 303: 1818-1822. 
Allende, D., S. A. Simon, et al. (2005). "Melittin-induced bilayer leakage depends on lipid material properties: evidence for toroidal pores." Biophys.J. 88(3): 1828-1837.

Armstrong, A. and S. L. Eck (2003). "EpCAM: A new therapeutic target for an old cancer antigen." Cancer Biol.Ther. 2(4):320-326.

Barrajon-Catalan, E., S. Fernandez-Arroyo, et al. (2010). "Cistaceae aqueous extracts containing ellagitannins show antioxidant and antimicrobial capacity, and cytotoxic activity against human cancer cells." Food and Chemical Toxicology 48: 2273-2282.

Barrajón-Catalán, E., M. P. Menéndez-Gutiérrez, et al. (2010). "Selective death of human breast cancer cells by lytic immunoliposomes: Correlation with their HER2 expression level." Cancer Letters 290: 192-203.

Bechinger, B. (1997). "Structure and functions of channel-forming peptides: magainins, cecropins, melittin and alamethicin." J.Membr.Biol. 156(3): 197-211.

Braun, S., F. Hepp, et al. (1999). "Monoclonal antibody therapy with Edrecolomab in breast cancer patients: Monitoring of elimination of disseminated cytokeratin-positive tumor cells in bone marrow." Clinical Cancer Research 5(12): 3999-4004.

Cimino, A., M. Halushka, et al. (2009). "Epithelial cell adhesion molecule (EpCAM) is overexpressed in breast cancer metastases." Breast Cancer Research and Treatment: 1-8.

Conrotto, P., C. Roesli, et al. (2008). "Identification of new accessible tumor antigens in human colon cancer by ex vivo protein biotinylation and comparative mass spectrometry analysis." International Journal of Cancer 123(12): 2856-2864.

Drummond, D. C., O. Meyer, et al. (1999). "Optimizing liposomes for delivery of chemotherapeutic agents to solid tumors." Pharmacol.Rev. 51: 691-743.

Elbayoumi, T. A. and V. P. Torchilin (2006). "Enhanced accumulation of long-circulating liposomes modified with the nucleosome-specific monoclonal antibody 2C5 in various tumours in mice: gamma-imaging studies." Eur. J. Nucl. Med. Mol. Imaging.

Gawronska, B., C. Leuschner, et al. (2002). "Effects of a lytic peptide conjugated to beta HCG on ovarian cancer: studies in vitro and in vivo." Gynecol.Oncol. 85(1): 45-52.

Haller, D. G. (2001). "Update of clinical trials with edrecolomab: A monoclonal antibody therapy for colorectal cancer." Seminars in Oncology 28(1 SUPPL. 1): 25-30.

Hansel, W., F. Enright, et al. (2006). "Destruction of breast cancers and their metastases by lytic peptide conjugates in vitro and in vivo." Mol.Cell Endocrinol. 260-262:183-189.

Hansen, C. B., G. Y. Kao, et al. (1995). "Attachment of antibodies to sterically stabilized liposomes: evaluation, comparison and optimization of coupling procedures." Biochim.Biophys.Acta. 1239(2): 133-144.

Hoskin, D. W. and A. Ramamoorthy (2008). "Studies on anticancer activities of antimicrobial peptides." Biochim.Biophys. Acta 1778(2): 357-75.

$\mathrm{Hu}, \mathrm{H} ., \mathrm{D}$. Chen, et al. (2006). "Target ability and therapy efficacy of immunoliposomes using a humanized antihepatoma disulfide-stabilized Fv fragment on tumor cells." J.Pharm.Sci. 95(1):192-199.

Ikegami, S., T. Tadakuma, et al. (2005). "Selective gene therapy for prostate cancer cells using liposomes conjugated with IgM type monoclonal antibody against prostate-specific membrane antigen." Human cell : official journal of Human Cell Research Society 18(1): 17-23.

Ikegami, S., K. Yamakami, et al. (2006). "Targeting gene therapy for prostate cancer cells by liposomes complexed with anti-prostate-specific membrane antigen monoclonal antibody." Human Gene Therapy 17(10): 997-1005.

Kirpotin, D., J. W. Park, et al. (1997). "Sterically stabilized anti-HER2 immunoliposomes: design and targeting to human breast cancer cells in vitro." Biochemistry 36(1): 66-75. 
Kirpotin, D. B., D. C. Drummond, et al. (2006). "Antibody targeting of long-circulating lipidic nanoparticles does not increase tumor localization but does increase internalization in animal models." Cancer Res. 66(13): 6732-6740.

Lin, J. H. and A. Baumgaertner (2000). "Stability of a melittin pore in a lipid bilayer: a molecular dynamics study." Biophys.J. 78(4): 1714-1724.

Lohner, K. and S. E. Blondelle (2005). "Molecular mechanisms of membrane perturbation by antimicrobial peptides and the use of biophysical studies in the design of novel peptide antibiotics." Comb.Chem. High Throughput Screen. 8(3): 241-56.

Madoz-Gúrpide, J., R. Kuick, et al. (2008). "Integral protein microarrays for the identification of lung cancer antigens in Sera that induce a humoral immune response." Molecular and Cellular Proteomics 7(2): 268-281.

Mamot, C., D. C. Drummond, et al. (2003). "Epidermal growth factor receptor (EGFR)targeted immunoliposomes mediate specific and efficient drug delivery to EGFRand EGFRvIII-overexpressing tumor cells." Cancer Res. 63(12): 3154-3161.

Maruyama, K., S. J. Kennel, et al. (1990). "Lipid composition is important for highly efficient target binding and retention of immunoliposomes." Proc.Natl.Acad.Sci.U.S.A. 87(15): 5744-5748.

McDermott, K. M., P. R. Crocker, et al. (2001). "Overexpression of MUC1 reconfigures the binding properties of tumor cells." International Journal of Cancer 94(6): 783-791.

Noble, C. O., D. B. Kirpotin, et al. (2004). "Development of ligand-targeted liposomes for cancer therapy." Expert Opinion on Therapeuty Targets 8(4): 335-353.

Olayioye, M. A., R. M. Neve, et al. (2000). "The ErbB signaling network: receptor heterodimerization in development and cancer." EMBO J. 19(13): 3159-67.

Pacakova, V. and K. Stulik (2000). "Validation of a method for determination of phospholipase A2 and melittin in bee venom preparations by capillary electrophoresis." J.AOAC Int. 83(3): 549-554.

Papadopoulos, I., E. Sivridis, et al. (2001). "Tumor angiogenesis is associated with MUC1 overexpression and loss of prostate-specific antigen expression in prostate cancer." Clinical Cancer Research 7(6): 1533-1538.

Papahadjopoulos, D., T. M. Allen, et al. (1991). "Sterically stabilized liposomes: improvements in pharmacokinetics and antitumor therapeutic efficacy." Proc.Natl.Acad Sci.U.S.A. 88: 11460-11464.

Park, J. W., K. Hong, et al. (2002). "Anti-HER2 immunoliposomes: enhanced efficacy attributable to targeted delivery." Clin.Cancer Res. 8: 1172-1181.

Park, J. W., K. Hong, et al. (1997). "Immunoliposomes for cancer treatment." Adv.Pharmacol. 40: 399-435.

Patrzykat, A. and S. E. Douglas (2005). "Antimicrobial peptides: cooperative approaches to protection." Protein Pep. Lett 12(19):19-25.

Perez-Paya, E., L. Braco, et al. (1991). "High-performance liquid chromatographic separation of modified and native melittin following transglutaminase-mediated derivatization with a dansyl fluorescent probe." J.Chromatogr. 548(1-2): 351-359.

Petrak, K. (2005). "Essential properties of drug-targeting delivery systems." Drug Discovery Today 10(23-24): 1667-1673.

Prang, N., S. Preithner, et al. (2005). "Cellular and complement-dependent cytotoxicity of Ep-CAM-specific monoclonal antibody MT201 against breast cancer cell lines." Br.J.Cancer. 92(2): 342-349.

Press, M. F., C. Cordon-Cardo, et al. (1990). "Expression of the HER-2/neu proto-oncogene in normal human adult and fetal tissues." Oncogene 5: 953-962. 
Punt, C. J. A., A. Nagy, et al. (2002). "Edrecolomab alone or in combination with fluorouracil and folinic acid in the adjuvant treatment of stage III colon cancer: A randomised study." Lancet 360(9334): 671-677.

Raghuraman, H. and A. Chattopadhyay (2007). "Melittin: a membrane-active peptide with diverse functions." Biosci.Rep. 27(4-5): 189-223.

Reichert, J. M., C. J. Rosensweig, et al. (2005). "Monoclonal antibody successes in the clinic." Nature Biotechnology 23(9): 1073-1078.

Saceda, M., C. Knabbe, et al. (1991). "Post-transcriptional destabilization of estrogen receptor mRNA in MCF-7 cells by 12-O-tetradecanoylphorbol-13-acetate." J.Biol.Chem. 266: 17809-17814.

Sadzuka, Y., K. Kishi, et al. (2003). "Effect of polyethyleneglycol (PEG) chain on cell uptake of PEG-modified liposomes." Journal of Liposome Research 13(2): 157-172.

Slamon, D. J., G. M. Clark, et al. (1987). "Human breast cancer: correlation of relapse and survival with amplification of the HER-2/neu oncogene." Science 235: 177-182.

Slamon, D. J., B. Leyland-Jones, et al. (2001). "Use of chemotherapy plus a monoclonal antibody against HER2 for metastatic breast cancer that overexpresses HER2." N. Engl. J. Med. 344(11):783-792.

Soman, N. R., S. L. Baldwin, et al. (2009). "Molecularly targeted nanocarriers deliver the cytolytic peptide melittin specifically to tumor cells in mice, reducing tumor growth." Journal of Clinical Investigation 119(9): 2830-2842.

Son, D. J., J. W. Lee, et al. (2007). "Therapeutic application of anti-arthritis, pain-releasing, and anti-cancer effects of bee venom and its constituent compounds." Pharmacol.Ther. 115(2): 246-70.

Sui, S. F., H. Wu, et al. (1994). "Conformational changes of melittin upon insertion into phospholipid monolayer and vesicle." J.Biochem. 116: 482-487.

Sznol, M. and J. Holmlund (1997). "Antigen-specific agents in development." Seminars in Oncology 24(2): 173-86.

Trzpis, M., P. M. McLaughlin, et al. (2007). "Epithelial cell adhesion molecule: more than a carcinoma marker and adhesion molecule." Am. J. Pathol. 171:386-395

Villari, V. and N. Micali (2008). "Light scattering as spectroscopic tool for the study of disperse systems useful in pharmaceutical sciences." Journal of Pharmaceutical Sciences 97(5): 1703-1730.

Wenqi, D., W. Li, et al. (2009). "EpCAM is overexpressed in gastric cancer and its downregulation suppresses proliferation of gastric cancer." Journal of Cancer Research and Clinical Oncology 135(9): 1277-1285.

Went, P. T., A. Lugli, et al. (2004). "Frequent EpCam protein expression in human carcinomas." Hum.Pathol. 35(1): 122-128.

Winkler, J., P. Martin-Killias, et al. (2009). "EpCAM-targeted delivery of nanocomplexed siRNA to tumor cells with designed ankyrin repeat proteins." Molecular Cancer Therapeutics 8(9): 2674-2683.

Yang, T., M. K. Choi, et al. (2007). "Antitumor effect of paclitaxel-loaded PEGylated immunoliposomes against human breast cancer cells." Pharm.Res. 24(12): 2402-11.

Yuji Yamamoto, M. Y., Mitsunori Sato, Koichi Sato, Satoshi Kikuchi, Hiroki Sugishita, Jun Kuwabara, Yusuke Matsuno, You Kojima, Masamitsu Morimoto, Atsushi Horiuchi, Yuji Watanabe (2011 Jan). "Feasibility of tailored, selective and effective anticancer chemotherapy by direct injection of docetaxel-loaded immunoliposomes into Her2/neu positive gastric tumor xenografts." Int. J. Oncology 38(1): 33-39.

Zuba-Surma, E. K., M. Kucia, et al. (2007). "The ImageStream System: a key step to a new era in imaging." Folia Histochem. Cytobiol. 45(4):279-290. 


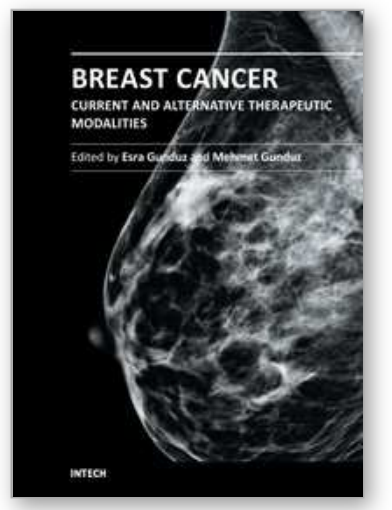

\author{
Breast Cancer - Current and Alternative Therapeutic Modalities \\ Edited by Prof. Esra Gunduz
}

ISBN 978-953-307-776-5

Hard cover, 540 pages

Publisher InTech

Published online 09, November, 2011

Published in print edition November, 2011

Cancer is the leading cause of death in most countries and its consequences result in huge economic, social and psychological burden. Breast cancer is the most frequently diagnosed cancer type and the leading cause of cancer death among females. In this book, we discussed various therapeutic modalities from signaling pathways through various anti-tumor compounds as well as herbal medicine for this deadly cancer. We hope that this book will contribute to the development of novel diagnostic as well as therapeutic approaches.

\title{
How to reference
}

In order to correctly reference this scholarly work, feel free to copy and paste the following:

Enrique Barrajón-Catalán, María P. Menéndez-Gutiérrez, Alberto Falcó, Miguel Saceda, Angela Catania and Vicente Micol (2011). Immunoliposomes: A Multipurpose Strategy in Breast Cancer Targeted Therapy, Breast Cancer - Current and Alternative Therapeutic Modalities, Prof. Esra Gunduz (Ed.), ISBN: 978-953-307-776-5, InTech, Available from: http://www.intechopen.com/books/breast-cancer-current-and-alternative-therapeuticmodalities/immunoliposomes-a-multipurpose-strategy-in-breast-cancer-targeted-therapy

\section{INTECH}

open science | open minds

\section{InTech Europe}

University Campus STeP Ri

Slavka Krautzeka 83/A

51000 Rijeka, Croatia

Phone: +385 (51) 770447

Fax: +385 (51) 686166

www.intechopen.com

\section{InTech China}

Unit 405, Office Block, Hotel Equatorial Shanghai

No.65, Yan An Road (West), Shanghai, 200040, China

中国上海市延安西路65号上海国际贵都大饭店办公楼 405 单元

Phone: +86-21-62489820

Fax: +86-21-62489821 
(C) 2011 The Author(s). Licensee IntechOpen. This is an open access article distributed under the terms of the Creative Commons Attribution 3.0 License, which permits unrestricted use, distribution, and reproduction in any medium, provided the original work is properly cited. 